\title{
Intensity modulated radiotherapy versus volumetric modulated arc therapy in breast cancer: A comparative dosimetric analysis
}

\author{
KR Muralidhar1, Bhudevi Soubhagya ${ }^{2}$, Shabbir Ahmed ${ }^{2}$ \\ ${ }^{I}$ Department of Radiation Physics, American Oncology Institute, Hyderabad, India \\ ${ }^{2}$ Basavataram Indo-American Cancer Institute and Research center, Hyderabad, India
}

Received December 09, 2014; Revised February 10, 2015; Accepted February 17, 2015; Published Online March 23, 2015

\section{Technical Report}

\begin{abstract}
Purpose: Intensity modulated radiotherapy (IMRT) and volumetric modulated arc therapy (VMAT) has the capacity to optimize the dose distribution. We analyzed the dosimetric differences of plans in treatment planning system (TPS) between VMAT and IMRT in treating breast cancer. Methods: Fourteen patients were simulated, planned, and treated with VMAT using single, double or partial arcs. IMRT treatments were generated using 4 to 5 tangential IMRT fields for the same patients. All treatment plans were planned for $50 \mathrm{~Gy}$ in 25 fractions. The VMAT and IMRT plans were compared using the planning target volume (PTV) dose and doses to the other organs at risk (OARs). Results: For the PTV, comparable minimum, mean, maximum, median, and modal dose as well equivalent sphere diameter of the structure (Equis) were observed between VMAT and IMRT plans and found that these values were significantly equal in both techniques. The right lung mean and modal doses were considerably higher in VMAT plans while maximum value was considerably lower when compared with IMRT plans. The left lung mean and modal doses were higher with VMAT while maximum doses were higher in IMRT plans. The mean dose to the heart and maximum dose to the spinal cord was lower with IMRT. The mean dose to the body was higher in VMAT plans while the maximum dose was higher in IMRT plans. Conclusion: Four field tangential IMRT delivered comparable PTV dose with generally less dose to normal tissues in our breast cancer treatment study. The IMRT plans typically had more favourable dose characteristics to the lung, heart, and spinal cord and body dose when compared with VMAT. The only minor advantage of VMAT for breast cases was slightly better PTV coverage.
\end{abstract}

Keywords: Breast Cancer; IMRT; VMAT

\section{Introduction}

The number of patients with breast cancer treated with radiation therapy has increased in the past few years. Normal tissue toxicities can limit the advantage of this treatment modality. ${ }^{1-2}$ Standard tangential beams have resulted in excellent local control rates, low rates of cardiac and pulmonary complications, and excellent cosmetic results in the vast majority of patients. ${ }^{3-7}$ Three-dimensional conformal radiotherapy (3D-CRT) and intensity-modulated radiotherapy (IMRT) techniques improve the treatment delivery by improving the target volume coverage and minimizing dose to other organs at risk (OARs). ${ }^{8-18}$ With IMRT, it is possible to reduce the volume of the lung irradiated to full doses by tangential fields, and in left-sided cases, the heart can also be partially spared. Several publications on this topic have discussed advantages and disadvantages of IMRT versus volumetric modulated arc therapy (VMAT). ${ }^{19-21}$

The use of RapidArc or VMAT (Novalis Tx, Varian Medical Systems, Inc, Palo Alto, USA) is receiving increased attention as an advanced technique in radiotherapy for fast delivery treatment with improved dose distribution. In an effort to identify and characterize dosimetric differences between VMAT and IMRT techniques for the breast cancer, we analyzed the calculated dose characteristics of VMAT and simulated treatment plans of IMRT in 14 breast cancer patients. For the purpose of this analysis, we assumed similar mean dose within target produces similar tumor control with these two techniques.

\section{Methods and Materials}

Fourteen patients presented with breast cancer (eight left side and six right side). Twelve of these cases were intact breast and two cases were post mastectomy. Each patient was immobilized using full body Vaclok (IBA, Bahnhofstrasse, Schwarzenbruck, Germany) for simulation and subsequent treatment. Axial computed tomography (CT) images of $5 \mathrm{~mm}$ thickness were obtained on a Philips Brilliance big bore 16-slice CT simulator (Philips Health Care, DA Best, Netherlands). The data was then exported to the Eclipse work- 
station (Eclipse treatment planning system (TPS), version 8.6; Varian Medical Systems, Palo Alto, CA, USA) where normal tissue and tumor segmentation were performed through the use of the available contouring tools. The gross tumor volume (GTV) was defined using soft tissue window. The planning target volume (PTV) was created by a $0.5 \mathrm{~cm}$ axial and $1.0 \mathrm{~cm}$ longitudinal expansion of the GTV. The OARs including lungs, heart, and spinal cord were contoured.

The VMAT treatment plans were designed using partial, single or paired arcs in the Eclipse TPS to achieve optimal PTV coverage and minimal OAR dose. High definition multileaf collimation was optimized using beams-eye-view for each arc of every patient's plan. The plans were then normalized to $100 \%$ of the dose to the mean of the PTV. The prescription dose was $50 \mathrm{~Gy}$ in 25 fractions for all plans. The constraints for the OARs included maximum dose of $35 \mathrm{~Gy}$, 30Gy, and 25 Gy to spinal cord, heart, and lung, respectively. The VMAT treatments were planned using the analytic anisotropic algorithm (AAA), Modified Batho algorithm for tissue heterogeneity corrections and AAA field volume dose algorithm for ARC calculations. Partial ARC, full ARC, and dual ARCs were used for planning to yield the best target coverage possible.

For comparative analysis, simulated treatment plans using 4 to 5 tangential intensity modulated beams were generated in Eclipse TPS. The same PTV and OAR structures as those defined at the time of VMAT treatment were used for the IMRT planning. Beams were manually selected to maximize access to the target while minimizing exposure to adjacent normal tissues. All the plans were optimized to allow for $95 \%$ coverage of PTV. Calculated dose characteristics to the PTV, spinal cord, lungs, and heart were compared. A two-tailed paired t-test was used to compare the VMAT and IMRT techniques in respect of dose to target and normal structures with a significance declared for a $p<0.05$ (Table 1). Additionally, both the VMAT and IMRT plans were normalized to 200 cGy at isocenter for the QA purpose. Patient specific QA was done using $0.1 \mathrm{cc}$ ionization chamber in a small cylindrical phantom.

\section{Results}

The IMRT plans demonstrated significantly lower mean doses to OARs and equivalent doses to the PTV when compared with VMAT plans. Representative dose distributions between VMAT treatment plans and IMRT plans are presented in (Figure 1) and dose volume histograms are shown in (Figure 2).
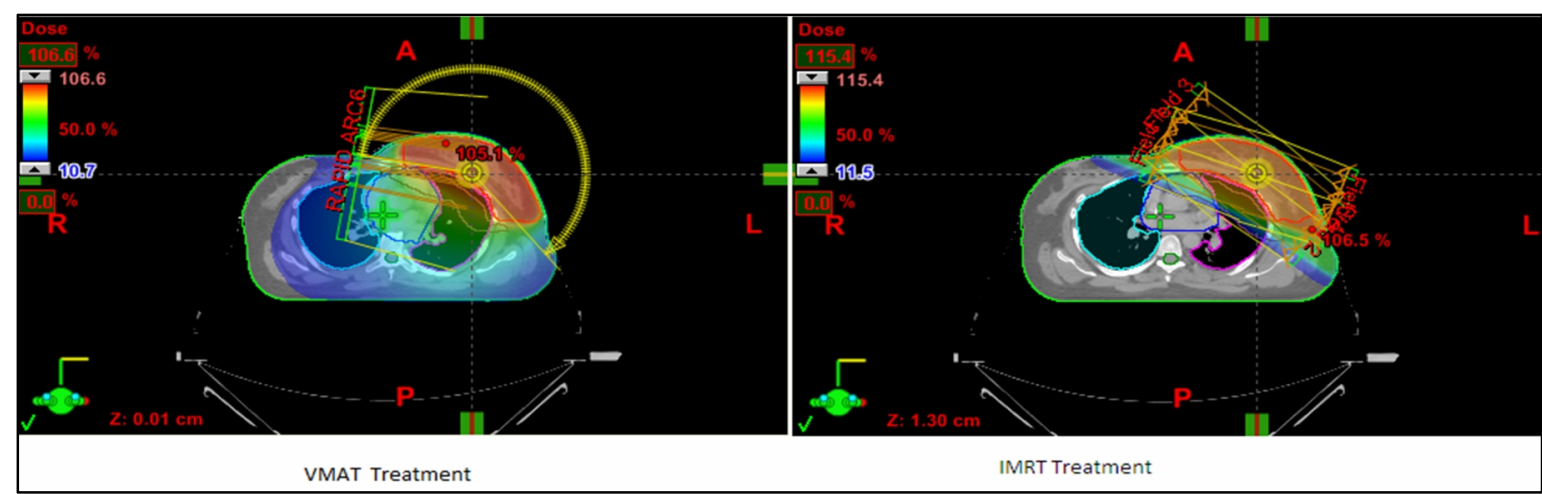

FIG. 1: An example of dose distribution between VMAT and IMRT breast cancer treatment plans

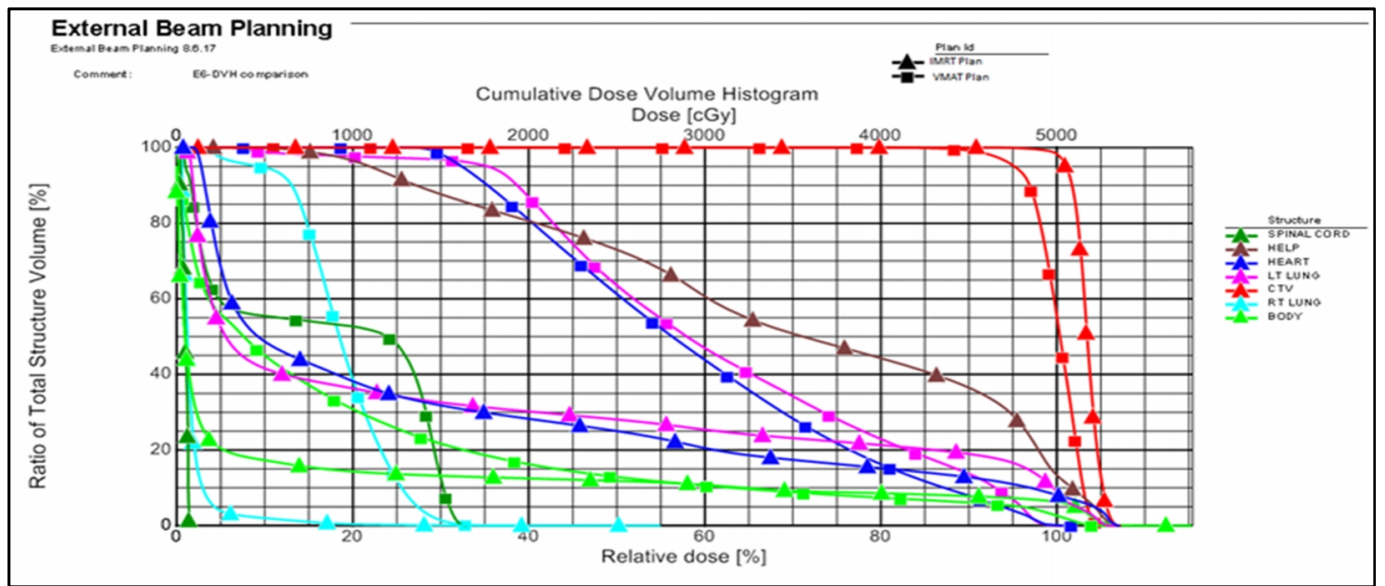

FIG. 2: An example of cumulative dose-volume histogram (DVH) of VMAT and IMRT plans for breast cancer. 
The IMRT plans exhibited equal minimum and mean doses for the PTV when compared to the VMAT plans. In comparison between the two plans, the PTV maximum doses were higher with in the IMRT plans than in the VMAT plans (Figure 3). Table 1 shows that the mean and median doses to the right lung were significantly lower with IMRT, whereas the maximum dose was significantly lower with VMAT.

IMRT delivery comparisons between two IMRT delivery techniques for QA plans are shown in Table 2. There is a significant difference in the mean values of dose range between IMRT and VMAT in case of lungs. The minimum and mean doses were higher in VMAT for the heart and spinal cord. While the heart maximum and spinal cord maximum doses are lower. The IMRT plan yields a significantly lower mean and median dose to the body (Table 1). For the VMAT, the Standard Deviation in Percentage (STD \%) is lower for the OARs and higher for the PTV with the exception of spinal cord. The maximum dose is higher in IMRT for all organs including the PTV. Equis is observed to be same for both techniques for target and the OARs. On average, the treatment MUs were 67\% less with VMAT compared with IMRT for the selected 14 patients in this study (Table 3 ).

\section{Discussion}

The VMAT technology is the most recent innovative technology in conventional photon therapy and widely used treatment technique in the entire world. As the treatment time, beam on time, and the number of monitor units (MUs) are less compared with IMRT, more hospitals prefer VMAT compared to other treatment techniques in photon therapy. The results provided in our study provide evidence that IMRT treatments can be developed that provide target coverage as VMAT.
According to Ashraf et al. ${ }^{22}$, the conformity to the PTV and critical structure sparing was better with 3DCRT than IMRT. In contrast to the Ashraf et al. ${ }^{22}$, Moorthy et al..$^{23}$ published that, with the use of IMRT technique, there was an improvement in CI when compared to CI of 3DCRT. The study conducted by Popescu et al. ${ }^{24}$ concluded that VMAT achieved similar PTV coverage and sparing of OARs, with fewer MUs and shorter delivery time than conventional IMRT. According to their study ${ }^{24}$, the healthy tissue volume percentages receiving 5 Gy were significantly larger with VMAT $(33.1 \% \pm$ $2.1 \%)$ and IMRT $(45.3 \% \pm 3.1 \%)$ than with conventional modified wide-tangent technique $(19.4 \% \pm 3.7 \%)$. According to study conducted by Johansen et al. ${ }^{25}$, a better homogeneity and conformation in PTV was observed in the VMAT plans. The highest minimum dose to PTV was observed in the conventional plans while no difference was observed for minimum significant doses D (98\%) and D (99\%) where D $(\mathrm{X} \%)$ is the dose received by $\mathrm{X} \%$ of the PTV volume. In terms of OAR sparing, the IMRT and RapidArc plans spare ipsilateral-lung better, but a $40 \%$ lower mean dose in the contra-lateral lung in the conventional plans is observed. The mean dose to the contra-lateral breast was lowest for the RapidArc plans as well as the V (10Gy) and the maximum dose.

RapidArc, however, is capable of producing better plans than IMRT for the test cases examined by Oliver et al. ${ }^{26}$ According to them, the conformity of dose distribution to target is better in RapidArc compared with IMRT. But integral dose is more in tomotherapy compared to RapidArc and IMRT. According to Nicolini et al. ${ }^{27}$, RapidArc showed dosimetric improvements with respect to IMRT. But they also have mentioned that mean dose to heart was $6.0 \pm 2.7 \mathrm{~Gy}$ (RapidArc) and $7.4 \pm$ 2.5 Gy (IMRT). We have also reported similar result in this study. We have demonstrated the pros and cons of the VMAT technique for breast cancer plans instead of just highlighting the only positive aspects of the VMAT.

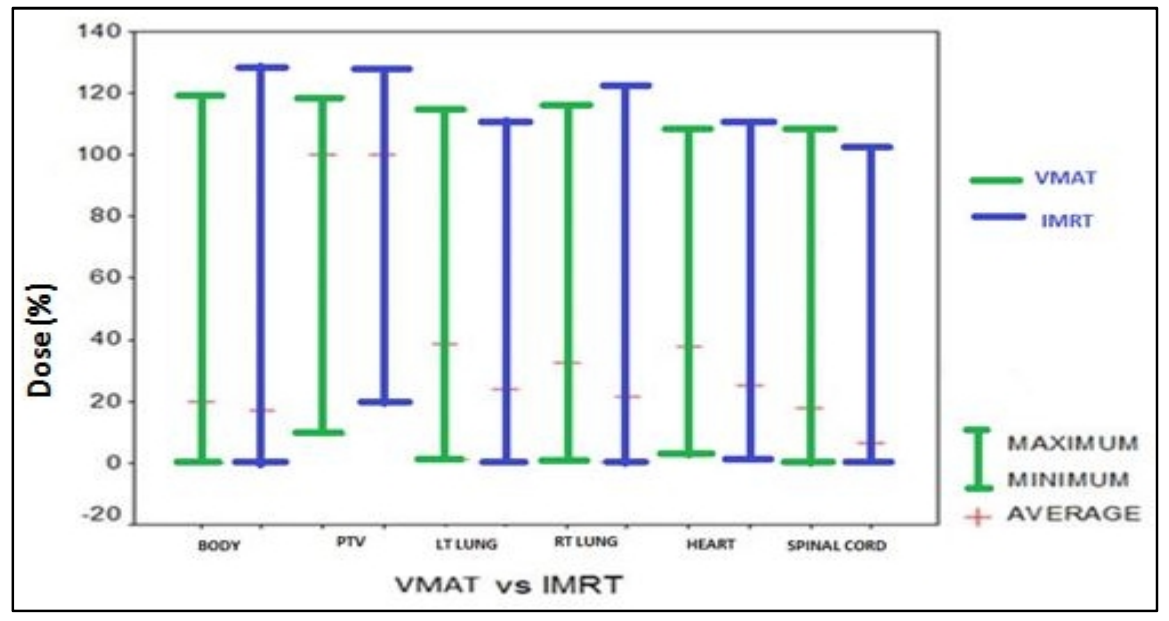

FIG. 3: Minimum, mean and maximum dose to PTV in VMAT and IMRT. 
TABLE 1: Dose calculations to PTV, body and normal structures with VMAT and tangential four field IMRT. The results are averaged over fourteen breast cancer cases in this study.

$[100 \%=$ prescribed dose; dose statistics obtained from the Eclipse TPS $]$

\begin{tabular}{|c|c|c|c|c|c|c|c|}
\hline & & \multicolumn{3}{|c|}{ VMAT (RapidArc) } & \multicolumn{3}{|c|}{$\underline{\text { IMRT }}$} \\
\hline & & Median & Range & $p$-value & Median & Range & $p$-value \\
\hline \multirow[t]{8}{*}{ PTV } & Dose coverage (\%) & 100 & 0 & 0.0001 & 100 & 0.4 & 0.0001 \\
\hline & Min value (\%) & 63.3 & 68.7 & 0.0001 & 58.6 & 62.5 & 0.0001 \\
\hline & Max value (\%) & 111 & 12.5 & 0.0001 & 114.7 & 20.8 & 0.0001 \\
\hline & Mean value (\%) & 100 & 0 & 0.0001 & 100 & 3.5 & 0.0001 \\
\hline & Modal D value (\%) & 101.1 & 5.8 & 0.0001 & 101 & 5.2 & 0.0001 \\
\hline & Median (\%) & 100.6 & 1.2 & 0.0001 & 100.4 & 4.3 & 0.0001 \\
\hline & STD (\%) & 4.6 & 3.5 & 0.0001 & 3.9 & 11 & 0.0001 \\
\hline & Equis (\%) & 12.9 & 7 & 0.0001 & 12.9 & 7 & 0.0001 \\
\hline \multirow[t]{8}{*}{ RT lung } & Dose coverage (\%) & 100 & 0 & 0.0001 & 100 & 0 & 0.0001 \\
\hline & Min value (\%) & 2.8 & 21.1 & 0.0026 & 0.6 & 4 & 0.0038 \\
\hline & Max value (\%) & 73.1 & 83.7 & 0.0001 & 93.7 & 103.9 & 0.0001 \\
\hline & Mean value (\%) & 18.7 & 59.7 & 0.0021 & 10.1 & 56.4 & 0.0021 \\
\hline & Modal D value (\%) & 14.9 & 86 & 0.0023 & 1.4 & 86 & 0.1324 \\
\hline & Median (\%) & 18.5 & 60.7 & 0.0001 & 9.4 & 63.9 & 0.0068 \\
\hline & STD (\%) & 7 & 23.8 & 0.0001 & 7.9 & 32.4 & 0.0004 \\
\hline & Equis (\%) & 12.4 & 1.8 & 0.0001 & 12.4 & 1.8 & 0.0001 \\
\hline \multirow[t]{8}{*}{ LT Lung } & Dose coverage (\%) & 100 & 0 & 0.0001 & 100 & 0 & 0.0001 \\
\hline & Min value (\%) & 2.5 & 15.6 & 0.0009 & 0.8 & 5.3 & 0.007 \\
\hline & Max value (\%) & 100.7 & 90 & 0.0001 & 102.5 & 90 & 0.0001 \\
\hline & Mean value (\%) & 48.1 & 49.6 & 0.0001 & 31.2 & 47.8 & 0.0003 \\
\hline & Modal D value (\%) & 21.9 & 93 & 0.0004 & 1.7 & 36.7 & 0.0522 \\
\hline & Median (\%) & 43.2 & 53.9 & 0.0001 & 7.8 & 43.7 & 0.0034 \\
\hline & STD (\%) & 24.7 & 26.7 & 0.0001 & 28.9 & 38.4 & 0.0001 \\
\hline & Equis (\%) & 11.5 & 2 & 0.0001 & 11.5 & 2 & 0.0001 \\
\hline \multirow[t]{8}{*}{ Heart } & Dose coverage (\%) & 100 & 0 & 0.0001 & 100 & 0 & 0.0001 \\
\hline & Min value (\%) & 7.7 & 17.2 & 0.0001 & 1.7 & 7.5 & 0.0006 \\
\hline & Max value (\%) & 101.1 & 29.4 & 0.0001 & 101.6 & 75.1 & 0.0001 \\
\hline & Mean value (\%) & 35.5 & 33 & 0.0001 & 25 & 41.6 & 0.0001 \\
\hline & Modal D value (\%) & 23.5 & 37.9 & 0.0001 & 2.5 & 32.7 & 0.0156 \\
\hline & Median (\%) & 31.4 & 39.3 & 0.0001 & 13.1 & 34.7 & 0.0001 \\
\hline & STD (\%) & 18.8 & 16.1 & 0.0001 & 22.5 & 32.7 & 0.0001 \\
\hline & Equis (\%) & 9.6 & 3 & 0.0001 & 9.6 & 3 & 0.0001 \\
\hline \multirow[t]{8}{*}{ SC } & Dose coverage (\%) & 100 & 0 & 0.0001 & 100 & 28.4 & 0.0001 \\
\hline & Min value (\%) & 0.7 & 18.4 & 0.0763 & 0.4 & 7.8 & 0.0658 \\
\hline & Max value (\%) & 11.6 & 77.8 & 0.0001 & 13.3 & 101 & 0.0035 \\
\hline & Mean value (\%) & 14.5 & 45.1 & 0.0001 & 2.4 & 43.4 & 0.0364 \\
\hline & Modal D value (\%) & 0.8 & 37.5 & 0.1176 & 1 & 18 & 0.0846 \\
\hline & Median (\%) & 15.1 & 41.5 & 0.0001 & 1.9 & 30.1 & 0.0389 \\
\hline & STD (\%) & 10.7 & 15.3 & 0.0001 & 1.1 & 29.3 & 0.0214 \\
\hline & Equis (\%) & 4.3 & 6.3 & 0.0001 & 4.3 & 6.3 & 0.0001 \\
\hline \multirow[t]{8}{*}{ BODY } & Dose coverage (\%) & 100 & 0 & 0.0001 & 96 & 33.9 & 0.0001 \\
\hline & Min value (\%) & 0 & 0.1 & 0.3343 & 0 & 0 & 0.0001 \\
\hline & Max value (\%) & 111.8 & 12.5 & 0.0001 & 120.9 & 19.3 & 0.0001 \\
\hline & Mean value (\%) & 18.5 & 14 & 0.0001 & 15.2 & 17.5 & 0.0001 \\
\hline & Modal D value (\%) & 0.6 & 1.7 & 0.0008 & 0.2 & 0.8 & 0.0028 \\
\hline & Median (\%) & 6.4 & 9.1 & 0.0001 & 1.2 & 4.6 & 0.0002 \\
\hline & STD (\%) & 27.3 & 10.9 & 0.0002 & 30.3 & 12.4 & 0.0001 \\
\hline & Equis (\%) & 34.4 & 8.3 & 0.0001 & 34.4 & 8.3 & 0.0001 \\
\hline
\end{tabular}

Dose coverage $(\%)=$ percentage of the structure volume covered by the dose matrix, which is a three dimensional matrix with in which the dose distribution is calculated; $p$-value (typically $\leq 0.05$ ) indicates strong evidence against the null hypothesis.

With the current beam arrangements calculations, it is reasonable to conclude that all of the analyzed IMRT approaches generally deliver fewer doses to adjacent normal tissues compared with VMAT without compromising target coverage. It was observed that a relatively larger volume of lung received a minimum dose and the prescribed dose limits with IMRT compared with VMAT. The absolute differences between mean doses were quite large approximately 17.5 Gy to 22 Gy. Body dose is more in VMAT when compared with IMRT treatments. 
TABLE 2: Comparisons between IMRT and VMAT delivery techniques.

\begin{tabular}{ccccc}
\hline $\begin{array}{c}\text { Patient } \\
\#\end{array}$ & $\begin{array}{c}\text { Dose in TPS at } \\
\text { isocenter (cGy) }\end{array}$ & $\begin{array}{c}\text { Dose with chamber } \\
\text { in IMRT (cGy) }\end{array}$ & $\begin{array}{c}\text { Dose with chamber in } \\
\text { VMAT (cGy) }\end{array}$ & $\begin{array}{c}\text { \% Variation Between } \\
\text { VMAT and IMRT }\end{array}$ \\
\hline 1 & 200 & 199 & 195 & 2 \\
2 & 200 & 197 & 203 & 2.9 \\
3 & 200 & 200 & 199 & 0.5 \\
4 & 200 & 198 & 200 & 1 \\
5 & 200 & 205 & 206 & 0.4 \\
6 & 200 & 203 & 195 & 3.9 \\
7 & 200 & 201 & 195 & 2.9 \\
8 & 200 & 201 & 201 & 0 \\
9 & 200 & 202 & 205 & 1.5 \\
10 & 200 & 203 & 205 & 1.5 \\
11 & 200 & 200 & 195 & 2.5 \\
12 & 200 & 201 & 202 & 0.5 \\
13 & 200 & 203 & 205 & 1.5 \\
14 & 200 & 198 & 201 & 1.5 \\
\hline \hline
\end{tabular}

TABLE 3: Monitor units (MUs) comparisons between IMRT and VMAT techniques.

\begin{tabular}{cccc}
\hline \hline $\begin{array}{c}\text { Patient } \\
\#\end{array}$ & $\begin{array}{c}\text { IMRT } \\
(\text { MU) }\end{array}$ & $\begin{array}{c}\text { VMAT } \\
(\text { MU) }\end{array}$ & $\begin{array}{c}\text { \% of variation between } \\
\text { VMAT and IMRT (MU) }\end{array}$ \\
\hline 1 & 2024 & 594 & 70 \\
2 & 420 & 240 & 43 \\
3 & 1236 & 417 & 66 \\
4 & 1334 & 283 & 79 \\
5 & 1135 & 502 & 56 \\
6 & 1050 & 181 & 83 \\
7 & 680 & 492 & 28 \\
8 & 2826 & 342 & 88 \\
9 & 1590 & 473 & 70 \\
10 & 1389 & 350 & 75 \\
11 & 1402 & 467 & 67 \\
12 & 1560 & 483 & 69 \\
13 & 1227 & 495 & 60 \\
14 & 2180 & 318 & 85 \\
\hline \hline
\end{tabular}

\section{Conclusion}

IMRT plans with four to five tangential beams provide comparable coverage of the PTV relative to VMAT plans in breast cancer. Typically comparable coverage was achieved by IMRT with less dose to adjacent normal tissue and integral dose. VMAT technique produced relatively larger volumes of lung, heart, and spinal cord exposed to the radiation.

\section{Conflict of interest}

The authors declare that they have no conflicts of interest. The authors alone are responsible for the content and writing of the paper.

\section{References}

1. Hurkmans CW, Borger JH, Bos LJ, et al. Cardiac and lung complication probabilities after breast cancer irradiation. Radiother Oncol 2000; $55: 145-51$.
2. Favourable and unfavourable effects on long-term survival of radiotherapy for early breast cancer: an overview of the randomised trials. Early Breast Cancer Trialists' Collaborative Group. Lancet 2000; 355:1757-70.

3. Potters L, Steinberg M, Wallner P, Hevezi J. How one defines intensity-modulated radiation therapy. Int J Radiat Oncol Biol Phys 2003; 56:609-10.

4. Glatstein E. The return of the snake oil salesmen. Int J Radiat Oncol Biol Phys 2003; 55:561-2.

5. Glatstein E. Intensity-modulated radiation therapy: the inverse, the converse, and the perverse. Semin Radiat Oncol 2002; 12:272-81.

6. Clarke DH, Le MG, Sarrazin D, et al. Analysis of local-regional relapses in patients with early breast cancers treated by excision and radiotherapy: experience of the Institut Gustave-Roussy. Int J Radiat Oncol Biol Phys 1985; 11:137-45.

7. Kestin LL, Sharpe MB, Frazier RC, et al. Intensity modulation to improve dose uniformity with tan- 
gential breast radiotherapy: initial clinical experience. Int J Radiat Oncol Biol Phys 2000; 48:1559-68.

8. Lo YC, Yasuda G, Fitzgerald TJ, Urie MM. Intensity modulation for breast treatment using static multi-leaf collimators. Int J Radiat Oncol Biol Phys 2000; 46:187-94.

9. Evans PM, Donovan EM, Partridge M, et al. The delivery of intensity modulated radiotherapy to the breast using multiple static fields. Radiother Oncol 2000; 57:79-89.

10. Li JG, Williams SS, Goffinet DR, et al. Breast-conserving radiation therapy using combined electron and intensity-modulated radiotherapy technique. Radiother Oncol 2000; 56:65-71.

11. Zackrisson B, Arevaran M, Karlsson M. Optimized MLC-beam arrangements for tangential breast irradiation. Radiother Oncol 2000; 54:209-212.

12. Donovan EM, Johnson U, Shentall G, et al. Evaluation of compensation in breast radiotherapy: A planning study sing multiple static fields. Int J Radiat Oncol Biol Phys 2000; 46:671-9.

13. Chang SX, Deschesne KM, Cullip TJ, et al. A comparison of different intensity modulation treatment techniques for tangential breast irradiation. Int $J$ Radiat Oncol Biol Phys 1999; 45:1305-14.

14. Smith MC, Li SD, Shostak CA, et al. Breast -conserving radiation therapy: Potential of inverse planning with intensity modulation. Radiology 1997; 203:871-6.

15. Hansen VN, Evans PM, Shentall GS, et al, Dosimetric evaluation of compensation in radiotherapy of the breast: MLC intensity modulation and physical compensators. Radiother Oncol 1997; 42:249-256.

16. Hong L, Hunt M, Chui C, et al. Intensity-modulated tangential beam irradiation of the intact breast. Int J Radiat Oncol Biol Phys 1999; 44:1155-64.

17. Carruther LJ, Redpath AT, Kunkler IH. The use of compensators to optimise the three dimensional dose distribution in radiotherapy of the intact breast. Radiother Oncol 1999; 50:291-300.

18. Kiricuta IC, Gotz U, Schwab F, et al. Target volume definition and target conformal irradiation technique for breast cancer patients. Acta Oncol 2000; 39:429-36.

19. Lee B, Lee S, Sung J, Yoon M. Radiotherapy-induced secondary cancer risk for breast cancer: 3D conformal therapy versus IMRT versus VMAT. J Radiol Prot 2014; 34:325-31.

20. Abo-Madyan Y, Aziz MH, Aly MM, et al. Second cancer risk after 3D-CRT, IMRT and VMAT for breast cancer. Radiother Oncol 2014; 110:471-6.

21. Jin GH, Chen LX, Deng XW, et al. A comparative dosimetric study for treating left-sided breast can- cer for small breast size using five different radiotherapy techniques: conventional tangential field, filed-in-filed, tangential-IMRT, multi-beam IMRT and VMAT. Radiat Oncol 2013; 8:89.

22. Ashraf M, Janardhan N, Bhavani P, et al. Dosimetric comparison of 3DCRT versus IMRT in whole breast irradiation of early stage breast cancer. Int J Cancer Ther Oncol 2014; 2:020318.

23. Moorthy S, Sakr H, Hasan S, et al. Dosimetric study of SIB-IMRT versus SIB-3DCRT for breast cancer with breath-hold gated technique. Int J Cancer Ther Oncol 2013; 1:010110.

24. Popescu CC, Olivotto IA, Beckham WA, et al. Volumetric modulated arc therapy improves dosimetry and reduces treatment time compared to conventional intensity-modulated radiotherapy for locoregional radiotherapy of left-sided breast cancer and internal mammary nodes. Int J Radiat Oncol Biol Phys 2010; 76:287-95.

25. Johansen S, Cozzi L, Olsen DR. A planning comparison of dose patterns in organs at risk and predicted risk for radiation induced malignancy in the contralateral breast following radiation therapy of primary breast using conventional, IMRT and volumetric modulated arc treatment techniques. Acta Oncol 2009; 48:495-503.

26. Oliver M, Ansbacher W, Beckham WA. Comparing planning time, delivery time and plan quality for IMRT, RapidArc and Tomotherapy. J Appl Clin Med Phys 2009; 10:3068.

27. Nicolini G, Clivio A, Fogliata A, et al. Simultaneous integrated boost radiotherapy for bilateral breast: a treatment planning and dosimetric comparison for volumetric modulated arc and fixed field intensity modulated therapy. Radiat Oncol 2009; 4:27. 